\title{
Identification of Hammerstein Systems using Triangular basis Functions
}

\author{
Khaled Elleuch \\ University of Sfax, National \\ Engineering School of Sfax, \\ Tunisia
}

\author{
Abdessattar Chaari \\ University of Sfax, National \\ Engineering School of Sfax, \\ Tunisia
}

\begin{abstract}
A new identification method is proposed for Hammerstein systems in presence of dead zone input nonlinearities. To describe and identify the nonlinear system, a new decomposition technique using the triangular basis functions is employed. Then a parameterized model is derived to represent the entire system. The approximation by Triangular basis functions for the description of the static nonlinear block conducts to a linear regressive model, so parameter matrices characterizing the considered model can be estimated. After this stage, Singular Values Decomposition (SVD) technique has been applied to separate the coupled parameters of matrixes. The numerical simulation results illustrate that the proposed approach can be a promising tool for identifying Hammerstein systems with dead zone nonlinearities.
\end{abstract}

\section{General Terms}

Modeling of nonlinear systems, Hammerstein systems, Triangular basis functions.

\section{Keywords}

Identification, SVD technique, Dead-zone nonlinearity.

\section{INTRODUCTION}

Modelling and identification of nonlinear dynamic systems constitute an essential stage in practical control design [1]. Indeed, several researchers published interesting works about this theme and many classes of nonlinear systems have been studied in the literature $[2,3]$. One of the studied classes is the block-oriented nonlinear systems having piecewise static nonlinearities [4, 5]. Among these models, we can cite particularly Hammerstein model [6, 7], Wiener model and Hammerstein-Wiener model [8, 9]. Hammerstein models with piecewise nonlinearities are frequently used in nonlinear systems control [10]. We find such nonlinearities in some actuator families such as dead zone nonlinearity, saturation nonlinearity, preload nonlinearity [11] etc.... In the literature, many methods of modelling and identification of Hammerstein systems have been proposed $[12,13]$. These methods have often used the decomposition based on the principle of "key term separation" leading to a form of model where the parameters of the linear and nonlinear blocks are separated [14]. This principle allows obtaining a model linear in parameters and the Recursive Least Square algorithm (RLS) can be applied [15], but the major disadvantage lies in the significant increase of the number of estimated parameters. To surmount this problem, we propose an approach which consists in building Hammerstein model having Piecewise nonlinear characteristics, such as for example the dead zone nonlinearity type. To describe the nonlinear system, we use a decomposition technique using the triangular basis functions to estimate the static nonlinear block. This technique leads to a particular form of Hammerstein model. This model is then formulated in a parametric form and regression analysis is used for improving the relationship between input and output signals. By recurring to SVD and RLS algorithm, optimal estimates of the parameters for matrices characterizing the considered model, can be determined.

\section{HAMMERSTEIN MODEL WITH DEAD ZONE NONLINEARITY}

\subsection{Description of block oriented nonlinear system}

We consider plants having the Hammerstein structure with ARX linear model shown in Fig 1.

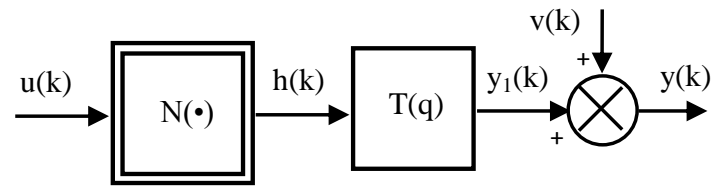

Fig 1: Hammerstein model

The signals $\mathrm{u}(\mathrm{k}), \mathrm{y}(\mathrm{k})$ and $\mathrm{y}_{1}(\mathrm{k})$ are, respectively, the model input, noisy model output and model output without noise, and the function $h(k)$ describing the nonlinear effects. The signal $\mathrm{h}(\mathrm{k})$ is a non available internal sequence related to the input only, which is defined by:

$\mathrm{h}(\mathrm{k})=\mathrm{N}(\mathrm{u}(\mathrm{k}))$

The transfer function $\mathrm{T}(\mathrm{q})$ of the linear block is described by

$\mathrm{T}(\mathrm{q})=\frac{\mathrm{B}\left(\mathrm{q}^{-1}\right)}{\mathrm{A}\left(\mathrm{q}^{-1}\right)}$

Where $\mathrm{q}^{-1}$ is the backward shift operator, and

$$
\begin{aligned}
& \mathrm{A}\left(\mathrm{q}^{-1}\right)=1+\mathrm{a}_{1} \mathrm{q}^{-1}+\ldots+\mathrm{a}_{\mathrm{n}_{\mathrm{A}}} \mathrm{q}^{-\mathrm{n}_{\mathrm{A}}} \\
& \mathrm{B}\left(\mathrm{q}^{-1}\right)=\mathrm{b}_{1} \mathrm{q}^{-1}+\mathrm{b}_{2} \mathrm{q}^{-2}+\ldots+\mathrm{b}_{\mathrm{n}_{\mathrm{B}}} \mathrm{q}^{-\mathrm{n}_{\mathrm{B}}}
\end{aligned}
$$

The noise of measure in the output $\mathrm{v}(\mathrm{k})$ is defined by

$$
\mathrm{v}(\mathrm{k})=\frac{1}{\mathrm{~A}\left(\mathrm{q}^{-1}\right)} \mathrm{e}(\mathrm{k})
$$


Where $\mathrm{e}(\mathrm{k})$ is a bounded measurement disturbance which is supposed to be a zero-mean, white noise sequence.

\subsection{Parameterization of static nonlinearity block}

The function $\mathrm{N}($.$) , describing the static nonlinear block, is$ supposed to be reminiscence within some given finite interval $\left[\mathrm{u}_{\min }, \mathrm{u}_{\max }\right]$. The signal $\mathrm{h}(\mathrm{k})$ is a non available internal sequence related to the input only. Then a decomposition of the nonlinear function can be used such as:

$$
\mathrm{N}(\mathrm{u})=\sum_{\mathrm{j}=1}^{\mathrm{p}} \mu_{\mathrm{j}} \eta_{j}(\mathrm{u})=\mu^{\mathrm{T}} \eta(\mathrm{u})
$$

Where $\mathrm{u}_{\min } \leq \mathrm{u} \leq \mathrm{u}_{\max }, \mu=\left[\mu_{1} \ldots \mu_{\mathrm{p}}\right]^{\mathrm{T}}$ is a parameter vector, $\eta(u)=\left[\eta_{1}(u) \ldots \eta_{p}(u)\right]^{T}$ and, $\eta_{j}() j=1,. \ldots p$, represents the $j^{\text {th }}$ basis function $\eta_{\mathrm{j}}($.$) . The basis functions are defined as follows$

$\eta_{j}(u)=\xi\left(\alpha_{j}\left(u-\gamma_{j}\right)\right)$

where $\alpha_{j}$ and $\gamma_{j}$ denote the dilation and translation parameters, respectively, and $\xi($.$) represents a generator$ function which belongs to a great family of functions containing Gaussian functions, generalized orthonormal basis functions [16], triangular functions, trigonometric functions etc ...

\section{IDENTIFICATION ALGORITHM}

The output of the nonlinear system $\mathrm{y}(\mathrm{k})$ can be formulated as:

$$
\mathrm{y}(\mathrm{k})=\left(1-\mathrm{A}\left(\mathrm{q}^{-1}\right)\right) \mathrm{y}(\mathrm{k})+\mathrm{B}\left(\mathrm{q}^{-1}\right) \mathrm{h}(\mathrm{k})+\mathrm{e}(\mathrm{k})
$$

Using (1), (3) and (4), the output can be given by the following equation:

$$
\begin{array}{rl}
y(k)=-a_{1} y(k-1)-\ldots-a_{n_{A}} & y\left(k-n_{n_{A}}\right)+b_{1} N(u(k-1)) \\
+\ldots+b_{n_{B}} N\left(u\left(k-n_{n_{B}}\right)+e(k)\right.
\end{array}
$$

In a matrix form, we can write the system output equation as follows

$$
\mathrm{y}(\mathrm{k})=\theta^{\mathrm{T}} \psi(\mathrm{k})+\mathrm{e}(\mathrm{k})
$$

Where $\theta$ is the parameter vector given by $\theta=\left[\begin{array}{ll}a & \theta_{b}\end{array}\right]^{\mathrm{T}}$, $\mathrm{a}=\left[\mathrm{a}_{1} \ldots \mathrm{a}_{\mathrm{n}_{\mathrm{A}}}\right]^{\mathrm{T}}, \quad \mathrm{b}=\left[\mathrm{b}_{1} \ldots \mathrm{b}_{\mathrm{n}_{\mathrm{B}}}\right]^{\mathrm{T}}, \quad \mu=\left[\mu_{1} \ldots \mu_{\mathrm{p}}\right]^{\mathrm{T}} \quad$ and $\theta_{b}=\operatorname{vec}\left(\mu b^{T}\right)$ with $\operatorname{vec}($.$) is an operator which stacks the$ columns of a matrix into a vector. The observation vector $\psi(\mathrm{k})$ is described by:

$$
\psi(\mathrm{k})=\left[\begin{array}{ll}
-\psi_{\mathrm{y}}(\mathrm{k}) & \psi_{\eta}(\mathrm{k})
\end{array}\right]^{\mathrm{T}}
$$

Where $\psi_{\mathrm{y}}(\mathrm{k})=\left[\mathrm{y}(\mathrm{k}-1) \ldots \mathrm{y}\left(\mathrm{k}-\mathrm{n}_{\mathrm{A}}\right)\right]^{\mathrm{T}}$

and $\psi_{\eta}(\mathrm{k})=\left[\eta(\mathrm{u}(\mathrm{k}-1)) \ldots \eta\left(\mathrm{u}\left(\mathrm{k}-\mathrm{n}_{\mathrm{B}}\right)\right)\right]^{\mathrm{T}}$
The estimated value of the parameter vector $\theta$ can be obtained by minimizing the following criterion

$\hat{\theta}=\arg \min \|\mathrm{Y}-\psi \theta\|_{2}$

Where $\mathrm{Y}=\left[\mathrm{y}\left(\mathrm{n}_{\mathrm{A}}\right) \ldots \mathrm{y}(\mathrm{r})\right]^{\mathrm{T}}$ and $\psi=\left[\psi^{\mathrm{T}}\left(\mathrm{n}_{\mathrm{A}}\right) \ldots \psi^{\mathrm{T}}(\mathrm{r})\right]^{\mathrm{T}}$ in the couples of input-output measurements $[\mathrm{u}(\mathrm{k}) ; \mathrm{y}(\mathrm{k})], \mathrm{k}=1, \ldots, \mathrm{r}$ where $r>n_{A}$.

The Recursive Least Square can be formulated by: $\hat{\theta}(\mathrm{k})=\hat{\theta}(\mathrm{k}-1)+\mathrm{P}(\mathrm{k}) \psi(\mathrm{k}) \varepsilon(\mathrm{k})$

$\left\{\begin{array}{l}\mathrm{P}(\mathrm{k})=\left[\mathrm{P}(\mathrm{k}-1)-\frac{\mathrm{P}(\mathrm{k}-1) \psi(\mathrm{k}) \psi^{\mathrm{T}}(\mathrm{k}) \mathrm{P}(\mathrm{k}-1)}{1+\psi^{\mathrm{T}}(\mathrm{k}) \mathrm{P}(\mathrm{k}-1) \psi(\mathrm{k})}\right] \\ \varepsilon(\mathrm{k})=\mathrm{y}(\mathrm{k})-\hat{\theta}^{\mathrm{T}}(\mathrm{k}-1) \psi(\mathrm{k})\end{array}\right.$

where $\varepsilon(k)$ is the prediction error, and $P(k)$ designates the adaptation matrix.

The separation of coupled parameters can be obtained by computing the Singular Value Decomposition (SVD) of $\operatorname{vec}^{-1}\left(\hat{\theta}_{\mathrm{b}}\right)$ and obtain $\mathrm{u}_{1}, \mathrm{v}_{1}$ and $\sigma_{\max }\left(\operatorname{vec}^{-1}\left(\hat{\theta}_{\mathrm{b}}\right)\right)$, then we can write

$\hat{\mu} \hat{b}^{\mathrm{T}}=\sigma_{\max }\left(\operatorname{vec}^{-1}\left(\hat{\theta}_{\mathrm{b}}\right)\right) \mathrm{u}_{1} \mathrm{v}_{1}^{\mathrm{T}}$

Where $\sigma_{\max }(),. \mathrm{u}_{1}$ and $\mathrm{v}_{1}$ are respectively the maximum singular value, $\mathrm{u}_{1}$ the first left eigenvector of $\mathrm{vec}^{-1}$ and $\mathrm{v}_{1}$ the first right eigenvector of $\mathrm{vec}^{-1}$. Using (12), the expressions of $\hat{\mu}$ and $\hat{b}$ can be respectively expressed, by:

$\hat{\mu}=\beta \sigma_{\max }\left(\operatorname{vec}^{-1}\left(\hat{\theta}_{\mathrm{b}}\right)\right) \mathrm{u}_{1}$

$\hat{b}=\frac{1}{\beta} v_{1}$

Where $\beta$ is a non null parameter that can be arbitrary chosen.

The nonlinear function N(.) can be improved from the estimate

$\hat{\mathrm{N}}(\mathrm{u}(\mathrm{k}))=\hat{\theta}_{\mathrm{b}} \psi_{\eta}(\mathrm{k})$

To conduct the nonlinear function $\mathrm{N}($.$) to a linear regressed$ form, we use the triangular basis functions. In the goal of an easy computation, the input interval $\left[\mathrm{u}_{\min }, \mathrm{u}_{\max }\right]$ is repeatedly divided into $\mathrm{p}$ partitions separated by a placement of points $\left\{\mathrm{u}_{1} \ldots \mathrm{u}_{\mathrm{p}}\right\}$, such that $\mathrm{u}_{\min }<\mathrm{u}_{\mathrm{j}}<\mathrm{u}_{\max }, \mathrm{u}_{\min }=\mathrm{u}_{1}$ and $\mathrm{up}=\mathrm{u}_{\max }$ with $\Delta=\mathrm{u}_{\mathrm{j}}-\mathrm{u}_{\mathrm{j}-1}$. The unit triangular generator function $\xi($.$) is$ defined as:

$\xi(\mathrm{z})=\left\{\begin{array}{rr}1+\mathrm{z} & \text { if }-1 \leq \mathrm{z}<0 \\ 1-\mathrm{z} & \text { if } 0 \leq \mathrm{z}<1 \\ 0 & \text { if } \mathrm{z}<-1 \text { and } \mathrm{z}>1\end{array}\right.$

The dilation and translation parameters can be written respectively by: $\gamma_{\mathrm{j}}=\mathrm{u}_{1}+\mathrm{j} \Delta$ and $\alpha_{\mathrm{j}}=1 / \Delta$ and $\gamma_{\mathrm{j}}=\mathrm{u}_{1}+\mathrm{j} \Delta$ 


\section{SIMULATION RESULTS}

To illustrate the feasibility of the proposed method, a nonlinear dynamic system with discontinuous nonlinearities using the Hammerstein model was implemented and tested by means of MATLAB packages. Hammerstein system is simulated and the estimation of the parameters are carried out on the basis of input output records. The estimation of the model parameters were carried out on the basis of input and output records. The following example shows the parameters estimation process for the linear dynamic part, which is given by the subsequent recursive equation:

$$
\begin{aligned}
& y(k)=1.6961 y(k-1)-0.8651 y(k-2)+ \\
& 0.5895 h(k-1)+0.4701 h(k-2)
\end{aligned}
$$

The static discontinuous nonlinearity is described by the following equation:

$$
N(u)=\left\{\begin{array}{lr}
u+0.28 & u \leq-0.28 \\
0 & -0.28<u<0.28 \\
u-0.28 & u \geq 0.28
\end{array}\right.
$$

We choose input signal as a zero-mean, white noise sequence uniformly distributed between -1 and 1 . The Signal to Noise Ratio SNR (the square root of the ratio of output and noise variances) was chosen as: $\mathrm{SNR}=25$ and $\mathrm{SNR}=50$.

To test the estimation quality of the model, the mean square error (MSE) is computed. Indeed, the MSE values for the nonlinear identification method were calculated for two values of SNR, and the results are tabulated in table 2. The MSE is defined as

$$
\mathrm{MSE}=\frac{1}{\mathrm{~N}} \sum_{\mathrm{k}=1}^{\mathrm{N}}(\mathrm{y}(\mathrm{k})-\hat{\mathrm{y}}(\mathrm{k}))^{2}
$$

Where $\hat{\mathrm{y}}(\mathrm{k})$ the predicted output and $\mathrm{N}$ is is the number of samples used in the identification process.

The evolution curves, for the output, the estimate values and the static nonlinearity (for SNR equal to 25) are given, respectively, in Figure 3, Figure 4 and Figure 5.

\section{DISCUSSION}

The previous curves of estimates parameters, (Fig. 3), show a good convergence of the algorithm. Indeed, the estimate value converges to the true value quickly. The statistical average, for the estimate of parameters of the last twenty samples with various values of SNR (25 and 50), is given in Table1. We note that, for the increase of the SNR values, the estimates converge quickly, towards the true values. The estimation results, for this kind of model, have proved the efficiency of this method for an acceptable value of SNR. In fact, for the value of SNR equal to 50 , the proposed model can approximate well the dead zone nonlinearity (Fig. 5) and the global system (Fig. 4). The computed value of Mean Square Error, given in Table 2, shows that the quality of estimation becomes better if the value of SNR increases.

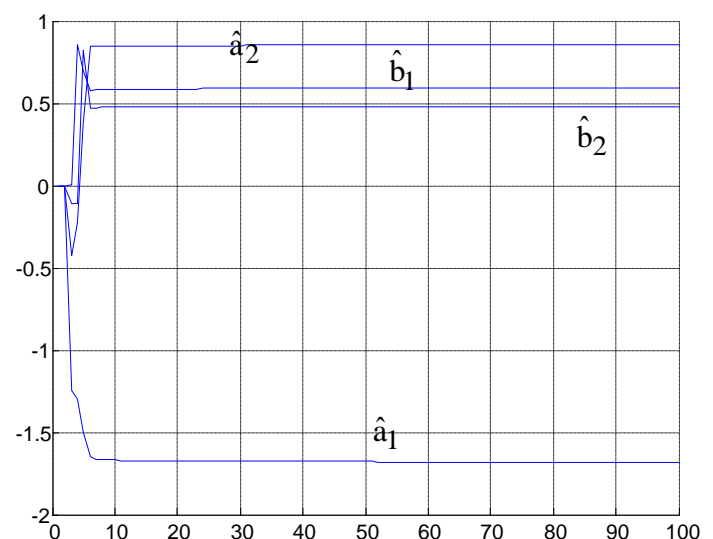

Fig 3: Parameter estimates.

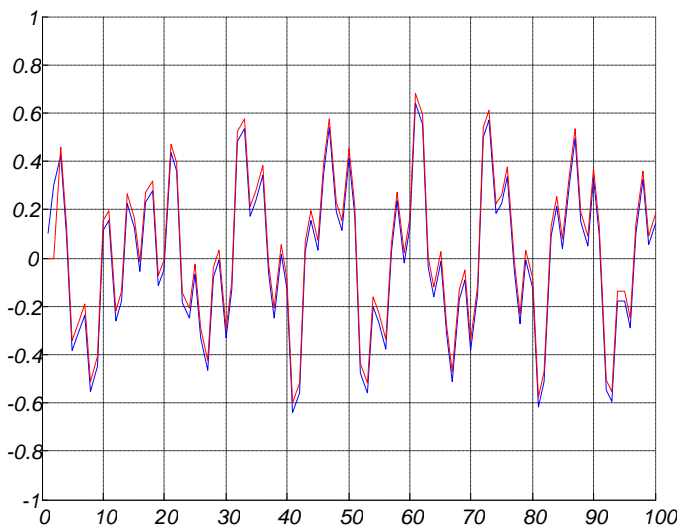

Fig 4: Real system response (blue) and identified model response (red).

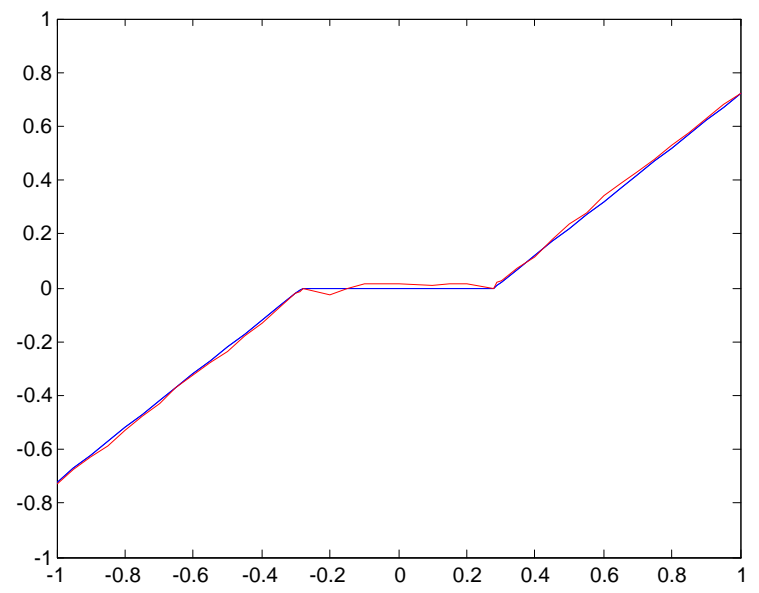

Fig 5: Static nonlinearity function: actual N(u) (blue) and estimated $\mathbf{N}(\mathbf{u})$ (red). 
Table 1. Estimate values of parameters

\begin{tabular}{|c|c|c|c|c|}
\hline SNR & $\hat{\mathrm{a}}_{1}$ & $\hat{\mathrm{a}}_{2}$ & $\hat{\mathrm{b}}_{1}$ & $\hat{\mathrm{b}}_{2}$ \\
\hline 25 & -1.6864 & 0.8561 & 0.5924 & 0.4773 \\
\hline 50 & -1.6959 & 0.8649 & 0.5893 & 0.4703 \\
\hline
\end{tabular}

Table 2. Mean square error

\begin{tabular}{|c|c|}
\hline SNR & MSE \\
\hline 25 & 0.0101 \\
\hline 50 & 0.0091 \\
\hline
\end{tabular}

\section{CONCLUSION}

In this paper, we have developed an identification algorithm for Hammerstein systems with dead zone input nonlinearities. The static nonlinearity block is approximated by triangular basis functions leading to a new description of Hammerstein model where a united parametric model can be built to describe the entire system. This method has permitted to obtain a linear regressed form; and therefore the least square techniques have been successfully used to estimate an oversized parameter matrix. The optimal estimates of the parameter matrix can be determined using SVD decomposition. It has been shown that the proposed model can approximate well the system and the estimation of the dead-zone curve converges to the true characteristic. The presented method can be easily extended to Hammerstein systems with other types of nonlinearities, e.g., preload nonlinearity, saturation nonlinearity, etc...

\section{REFERENCES}

[1] J. Wang, A. Sano, D. Shook, T. Chen, B. Huang 2007. A blind approach to closed-loop identification of Hammerstein systems. Int. J. Control 80 (2) 302-313.

[2] Bai E.W. 2002. Identification of linear systems with hard input nonlinearities of known structure", Automatica, vol. 38 , no. 5, pp. 853-860.

[3] Vörös J. 1997. Parameter Identification of Discontinuous Hammerstein Systems. Automatica 33 No. 6, 1141-1146.

[4] Kara T., and Eker I. 2004. Nonlinear modelling and identification of a DC motor for bidirectional operation with real time experiments. Energy Conversion and Management, vol. 45, pp. 1087-1106.

[5] T. H. van Pelt and D. S Bernstein 2001. Nonlinear system identification using Hammerstein and nonlinear feedback models with piecewise linear static maps. Int. J. Control, vol. $74, \mathrm{n}^{\circ} 18$, pp. 1807-1823.

[6] Khaled ELLEUCH and Abdessattar CHAARI 2010. Modelling and Estimation of Hammerstein System with Preload Nonlinearity. Leonardo Journal of Sciences (LJS), Issue 16 (9), p. 13-20.

[7] Vörös J. 2005. Identification of Hammerstein systems with time-varying piecewise-linear characteristics nonlinearities. Journal of electrical engineering, vol. 57, No. 1, pp. 42-46.

[8] Elleuch K., M. Kharrat, A. Chaari and M. Chaabane 2009. Modeling and identification of block-oriented heat transfer process. Int. J. of Information and Systems Sciences, vol. 5, $\mathrm{n}^{\circ} 1$, , pp. 41-56.

[9] D.Q. Wang, F. Ding 2008. Extended stochastic gradient identification algorithms for Hammerstein-Wiener ARMAX systems. Comput. Math. Appl. 56 (12) 31573164 .

[10] Tao G. and M. Tian 1998. Discrete-time adaptive control of systems with multisegment piecewise-linear nonlinearities. IEEE Trans. Autom. Control, vol. 43, no. 5, pp. 719-723.

[11] Kung, M. C. and Womack, B. F. 1984b. Discrete time adaptive control of linear systems with preload nonlinearity, Automatica 20, 477-479.

[12] Vörös J. 2002. Modelling and parameter identification of systems with multi segment piecewise-linear characteristics IEEE Transactions on Automatic Control, vol. AC 47, $\mathrm{N}^{\circ}$ 1, pp. 184-188.

[13] Billings S., S. Fakhouri. 1982. Identification of systems containing linear dynamic and static nonlinear elements. Automatica, pp. 15-26.

[14] Vörös J. 1999. Iterative algorithm for parameter identification of Hammerstein systems with two-segment nonlinearities. IEEE Transactions on Automatic Control (44). pp. 2145-2149.

[15] Vörös J. 2010. Recursive Identification of Systems with Noninvertible Output Nonlinearities. Informatica, Vol. 21, No. $1,139-148$.

[16] Gomez J.C., E. Baeyens 2004. Identification of blockoriented nonlinear systems using orthonormal bases. Journal of Process Control 14, pp. 685-69. 